\title{
Brain Tumor identification by Convolution Neural Network with Fuzzy C-mean Model Using MR Brain Images
}

\author{
Abd El Kader Isselmou, Guizhi Xu, Zhang Shuai, Sani Saminu, Imran Javaid, Isah Salim Ahmad \\ State Key Laboratory of Reliability and Intelligence of Electrical Equipment, Hebei University of \\ Technology, Tianjin, 300130, P.R. China.
}

Received: November 23. Revised: December 29, 2020. Accepted: December 29, 2020.

\begin{abstract}
Medical image computing techniques are essential in helping the doctors to support their decision in the diagnosis of the patients. Due to the complexity of the brain structure, we choose to use MR brain images because of their quality and the highest resolution. The objective of this article is to detect brain tumor using convolution neural network with fuzzy c-means model, the advantage of the proposed model is the ability to achieve excellent performance using accuracy, sensitivity, specificity, overall dice and recall values better than the previous models that are already published. In addition, the novel model can identify the brain tumor, using different types of MR images. The proposed model obtained accuracy with $98 \%$.
\end{abstract}

Keywords - MRI, CNN, FCM, Tumor Detection, accuracy, sensitivity and ndice.

\section{INTRODUCTION}

Abnormal cell development [1-2] is the leading cause of brain tumors. Glioma is a shocking brain tumor [3]. It is divided into four levels. Grades I and II are lower, while grades III and IV are higher. Glioblastoma is a grade 4 glioma, and ischemic stroke occurs due to the occurrence of a rare glioblastoma in the brain. Ischemic stroke is unstable and has undergone several stages divided into acute, subacute, and chronic. At all of these stages, cell destruction will occur, altering and expanding the molecular structure of brain tissue on MRI $[4,5]$. This is because automated tumor detection and treatment planning is a complicated task.

The technical differences and differences between semiautomatic and impressive automatic detection and classification technologies [6]. Important information and information related to regular information mutual assistance between normal and abnormal information [3, 10]. The difference between informatics and informatics [11], histogram and tension value [12]. The neuron convolutional neural network $(\mathrm{CNN})$ deployed a temporary tumour detector [13, 14]. CNN has added Mandarin [13], continuous [15] and professional [16-17] to distinguish the function of the IRM inverted certificate of IRM images, and in general, Edme, rebellion and non-rebellion.

The above methods provide better results in detecting high-grade glioma (HGG), but these methods show inferior performance in detecting low-grade glioma (LGG). The CNN method requires equipment with high computing power and storage efficiency [15]. Therefore, to solve this problem, a new lightweight four-layer LSTM model is proposed, which can provide better data processing in time. As a result, it is more useful for multi-sequence MRI learning.

Using high-resolution medical images can make it easier for doctors to diagnose diseases. Super Resolution (SR) is used to improve accuracy. In the literature, SR is used for medical images [16-17], MR images [18], MR images of the brain [19], and MR images of the heart [20] and fundus images [21]. Several methods have been used in the literature to detect and classify tumor regions using MR images automatically. Thad and others. [22] Constructed a hybrid system with fuzzy C-mean (FCM) growth area for tumor analysis. Sachdeva et al. $[23,24]$ used support vector machines (SVM) and functional neural network (ANN) machine learning models to classify brain tumors using genetic algorithms (GA) (GA-SVM and GA-ANN) in a hybrid architecture. Malek et al. [25] used a hybrid method of image compression for wavelet conversion and used the "Deep Wavelength Autoencodre" (DWA) tool to compress the images to process brain MRI images. In addition, a deep neural network (DNN) is used to classify images and used other conventional classification techniques to compare the performance of DWA-DNN models.

Zeng et al. [19] proposes model proposes single-contrast and multi-contrast ultra-precise reconstruction of artificial and real brain MRI images. Swati et al. [26] proposed a learningbased method that uses previously tested deep CNN models to 
learn to transfer brain MRI images. The accuracy of the proposed method after five verifications is $94.82 \%$ because it does not use any manual functions and requires minimal preprocessing. Deepak and Amir [27] used pre-trained GoogleNet to extract three types of brain from MR images of the brain through deep CNN. Selvapandian and Manivannan [28] used non-sub contour line transduction (NSCT) to improve brain images and then removed tissue features from the enhanced brain images .They used the adaptive neurofuzzy inference system (ANFIS) classification method to train and classify these extracted features. In their research, Pan et al. [29] used Two-dimensional MR images of the brain are used to compare the performance of CNN and RPNN algorithms. In addition, the kernel have received training at different levels.

In brain tumor segmentation, we found several methods to develop parametric or non-parametric probability models for basic data explicitly. These models usually include probability functions that match observations and older models. Because they are abnormal, tumors can be divided into outer parts of normal tissue, limited by shape and connectivity [30]. Other methods are based on the probability atlas [31]-[32]. For brain tumors, due to changes in the shape and location of the tumor, the atlas must be estimated during segmentation [31]-[32]. The tumor growth model can be used to estimate its huge effect and can be used to improve the atlas [33], [32]. The pixel neighbourhood provides useful information for achieving smoother segmentation through Markov Random Field (MRF) [31]. Zhou et el. [34] also used MRF to segment brain tumors after the first excessive image is increased to hyperopia, and perform map-based estimation based on probability functions. Minzi et al [34] also pointed out that in the absence of data, the generative model can generalize well, but it can transform the prior knowledge into a model that can be appropriately degraded.

Another type of method learns to distribute directly from the data. Throughout the training phase, Al is useful, and these methods can scan brain tumor patterns that do not follow a specific model. This method usually treats pixels as being independent and uniformly distributed [35], although context information can be presented through these functions. Therefore, some isolated pixels or small groups may be incorrectly classified as the wrong class, sometimes in locations that are not physiologically and anatomically probable. To work around this problem, some authors include neighbourhood information by including a predictor of the likelihood of the classifier in the region. The conditional random domain [35] - [36]. Farzam Kharajinezhadian et al [40] proposed method based on verification though a color image of the palm of the hand with index, middle, Ring and Little fingers and implemented a new method for extracting texture features in images from 177 people from Hong Kong Polytechnic University. Contactless 3D / 2D image database is another proposed feature extraction method consists in using revolutions in each of the Gabor filters applied in different directions and scales each of the RGB components of the images individually. Before classifying binary genetics, the algorithm is applied to use the best combination of features for each color component. The system performance was examined in unimodal and multimodal mode using SVM classification and the best result is obtained based on combining the properties of the palm and four fingers with an EER of $0.67 \pm$ 0.13 .

Mehidi. A et al. [41] proposed a method, which focus on automation Micro calcifications detection through mammographic image processing. it helps Experts in their work because the number of pictures to inspect and rated is very large. An automated system for this purpose is an algorithm for the detection of micro calcification clusters that is proposed according to a certain methodology. The first is mammography pretreated with a technique that improves quality Mammography (improvement of local contrast). Then the clusters are identified by means of stochastic analysis based on the hidden Markov Channels with Hilbert-Peano analysis of medical images. This can be the detection of node components, such as Micro-calcifications with precision by entering size information. The results obtained are very clear visually, precise and show that the proposed approach enables a successful extraction of Micro calcifications from referential mammography images of the VIES database. We have also shown that the use of recognize hidden Markov Chains (HMM) are more efficiently Micro calcifications, as HMMs are mainly spatial regularity constraint for image processing. Based on the objective performance measurements, namely the True Positive Rate (TPR) and the false positive rate (FPR) that the comparative study conducted in the three breast densities have shown the effectiveness of our method regardless of the type of breast density.

In this article, we proposed a supervised model based on $\mathrm{CNN}$ algorithm with fuzzy c-mean and efficient brain tumor detection. We produced an excellent overall performance using different values..

\section{MEthodology}

In this proposed model, the methodology is consists of six phases, the first phase is input MRI data that includes original MR brain images, the second phase is applied pre-process to remove the noise and clean the data, the third phase used fuzzy c-mean to segment the MR images, the fourth phase used convolution neural network to identify the tumor area in $\mathrm{MR}$ brain images, the fifth phase detected tumor only, while the sixth phase is the performance of CNN with FCM modelbased on accuracy, sensitivity, specificity, overall dice and recall value.figure1 explain more about the proposed model. 


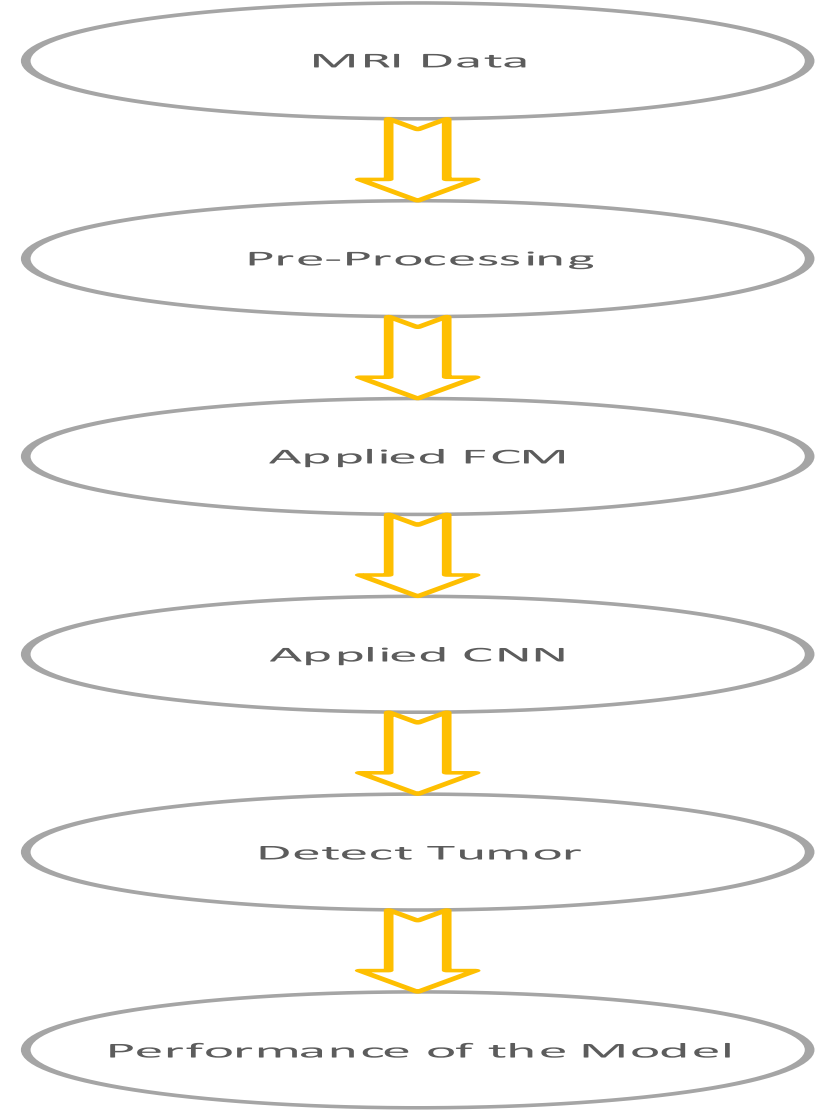

Fig.1 Architecture of proposed CNN-FCM Model.

\section{A. Pre-processing}

The pre-processing process is an important work in image processing, as the program can improve the image information, thus suppressing confusion or upgrade some of the most important images for a more accurate preparation, Many calculations and systems have been proposed and obtained as reported in [37] [38].

Due to its high sensitivity to high frequencies, image-denoising using Gaussian filters is widely used in medical image processing. The definition of the Gaussian filter is as follows:

$$
G(x, y)=\frac{1}{2 \pi \sigma^{2}} \exp -\frac{x^{2}+y^{2}}{2^{\sigma^{2}}}
$$

Where $\sigma$ the standard deviation of the Gaussian distribution, is For Skull stripping in BMRI images, many methods are proposed.

\section{B. Fuzzy C-Means}

The Fuzzy C-Means (FCM) algorithm is a collection of systems created by Dunn and Bezdek; it is categorized by the additional titration of Mathieu Patitucci's voxel groups (data) with magnetic resonance (MR) brain images. And the clustering is specified by the PSO algorithm $\left(\mathrm{x}^{\wedge} \rightarrow\right)(\mathrm{t}+1)$, the updated position vector of the particle (voxel or pixel) derived by the PSO algorithm serves as the centroid value, and based on this value, the number of clusters is used in the FCM algorithm. The algorithm also uses PSO allocation to determine the center of the member row and cluster duplication to limit the proposal and voxel grouping function.

$$
l_{k}=\sum_{i=j}^{N} \sum_{j=1}^{C} \delta_{i j}\left\|X_{i}-C_{j}\right\|^{2}
$$

$N$ Describes the number of incoming data points as input to the algorithm and $K$ shows the number of iterations to be performed.

\section{Convolution Neural Network}

Pattern recognition is a new feature of CNNs, which has performed remarkably well in detecting patterns in images of various types [39]. A typical CNN consists of input, wrapping, maximum clustering, fully connected layers (FC), and output layers stacked hierarchically. The $\mathrm{CNN}$ core is a filter of various shapes like $3 \times 3,7 \times 7$ and $13 \times 13$, which is wrapped over the inputs in a sliding window manner to create a feature map. A feature map is a collection of features organized into an organized structure, where each point on the map is connected to the output of the previous layer through weights. By increasing, the size of the nucleus, the neighbourhood area increases, and more contextual information is added to the function map in the form of neuronal activation. Faj Feature map is calculated as in the equation (3):

$$
F_{a j}=b_{a}+\sum_{j} K_{a j} * I_{j}
$$

Where $b_{a}, K_{a_{j} j}$ and $I_{j}$ are the bias term, convolution kernel and input plane, respectively, whereas * it represents the convolution operation.

\section{D.Accuracy value}

Accuracy and reliability are also called hash precision; it is used to determine the validity of the variables used to evaluate the hash algorithm. The accuracy is expressed by formula (4).

$$
\text { Accuracy }=\frac{\alpha+\beta}{\alpha+\beta+\gamma+\beta}
$$

$\propto \& \beta$ describes the true positive and true negative respectively.

$\gamma \& \xi$ denotes the false positive and false negative individually.

\section{E.Dice Overlap Index (DOI)}

It is represented by the value of the Jaccard J index (A, B). The DOI specifies the purpose of the overlap of the input image (A) and the final segmented image (B). The DOI states the calculation in equation (5).

$$
D(A, B)=2 \times \frac{\pi(A B)}{1+\pi\left(A_{0} B\right)}
$$

\section{A. F. Sensitivity}

The sensitivity value indicates the appropriate division or classification in the input image, in addition to determining the efficiency of accurate identification of the tumor region as well as other tissue regions. This is explained in equation (6).

$$
T P R=\frac{\alpha}{\alpha+\xi}
$$




\section{B. G. Specificity}

The specificity defines a specific word or algorithm used to classify the various parts of the normal tissue present in the area into the input image capacitance, and the specificity is shown in equation (7).

$$
\text { TNR }=\frac{\beta}{\beta+\gamma}
$$

H. Recall

$$
\text { Recall }=\frac{T P}{T P+F N}
$$

\section{M ATERIALS}

In this work, we have taken the MR brain database from Kaggle site.BRATS2013, the database consists of 150 MR brain images with tumor, $100 \mathrm{MR}$ brain images without tumor as shown in figures. 2 and 3 show it.

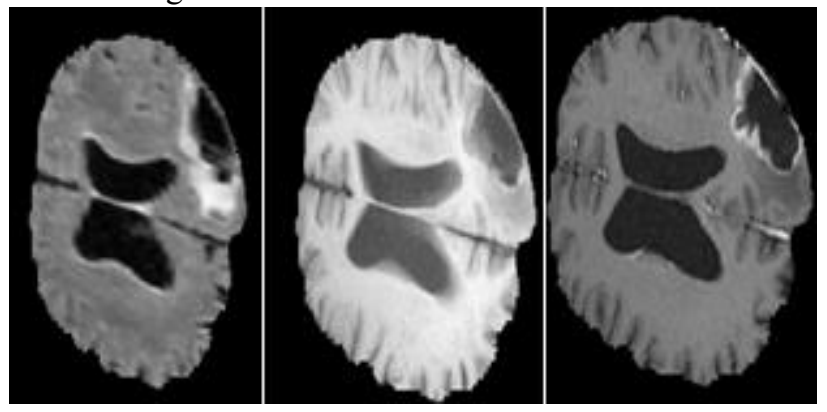

Fig.2 MRI brain Images with Tumor.

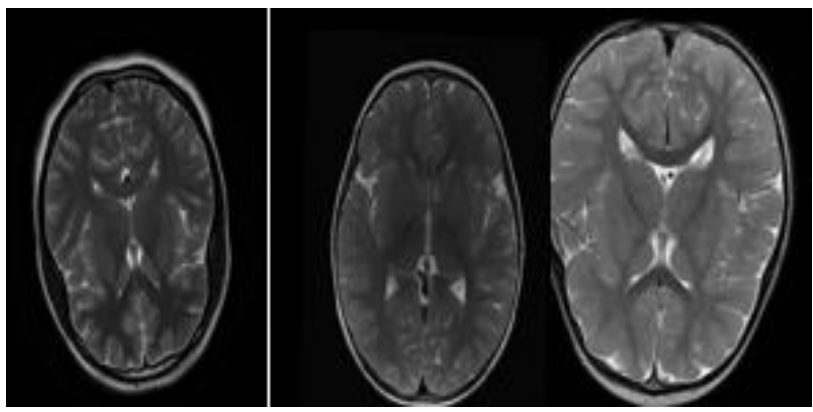

Fig.3. MRI Brain Images without Tumor.

\section{RESULTS AND DISCUSSION}

\section{A. Results of Brain Tumor Detection Using CNN-FCM Model}

Figure. 4 and 5 Shows that the model CNN-FCM can identify tumor area and detect it alone using different types of MR brain images.

Images (a): described MR original Images

Images (b): described Tumor Area using CNN-FCM model Images ( $)$ : described Tumor detected using CNN-FCM model.

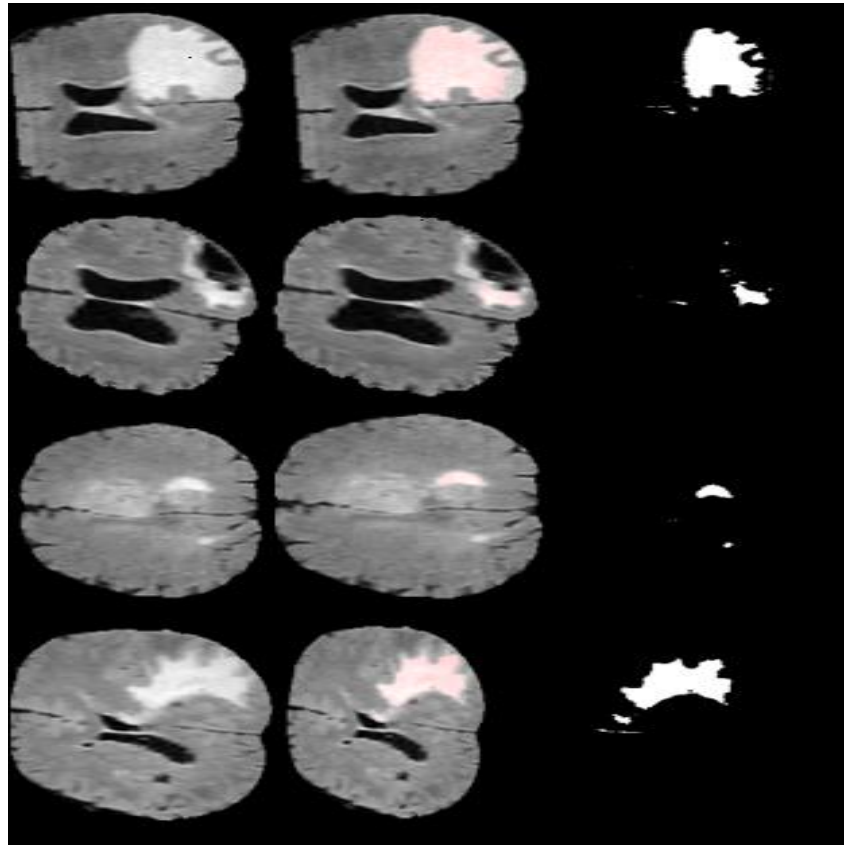

Fig.4. MRI Brain FLAIR Images Processed Using CNN-FCM Model.

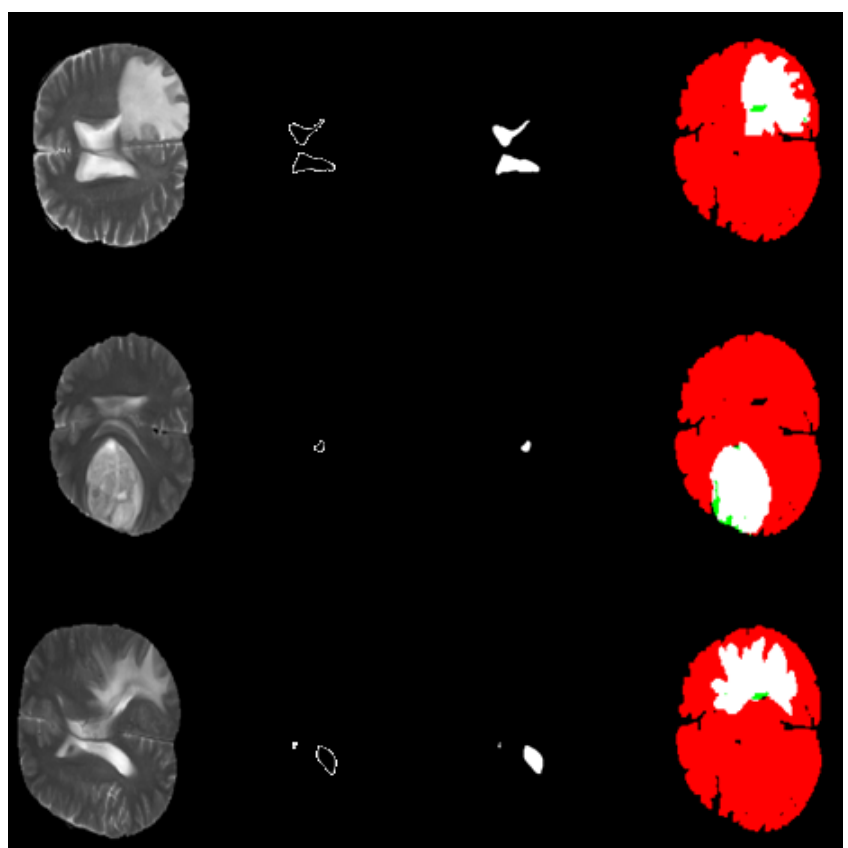

Fig.5. MRI Brain T1-weight Images Processed Using CNN-FCM Model.

\section{B. The Results of Performance of the CNN-FCM Model}

The method utilized in this article, which uses CNN-FCM model on BRATS2013 data is compared with the performance of ANN, SVM and DNN models, the comparison is based on accuracy, sensitivity, specificity, overall dice and recall values. The entire previous model using tumor classification on MR brain images. 


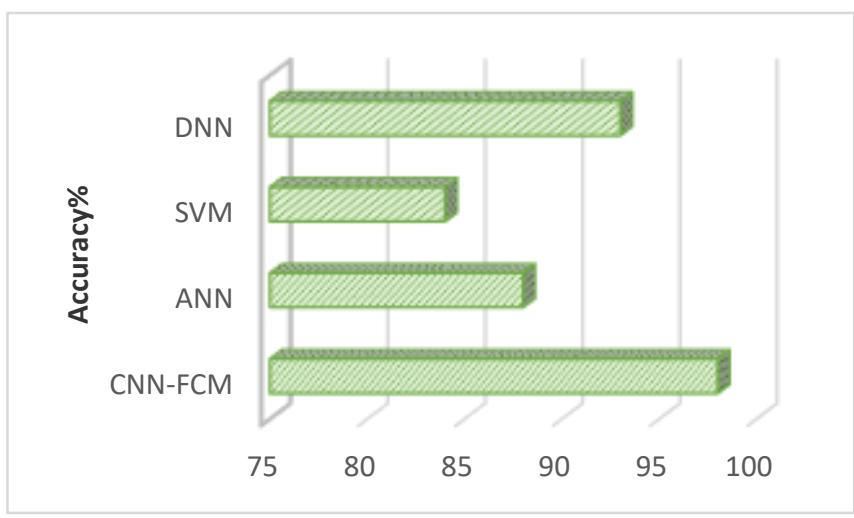

Fig.6. comparison between the CNN-FCM Model with previous models using accuracy value.

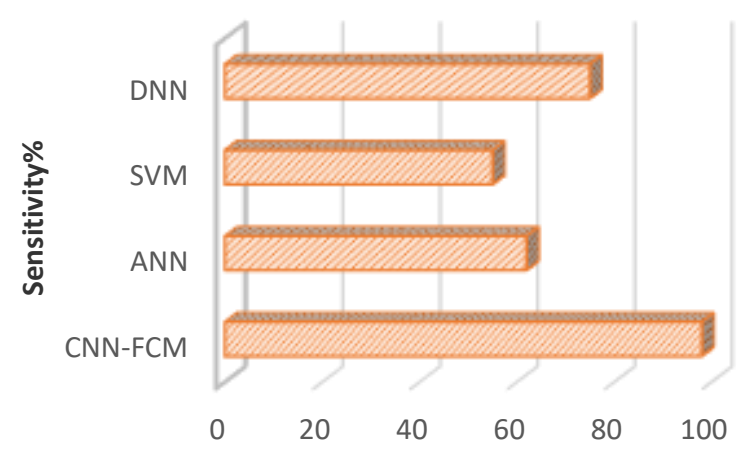

Fig.7.Comparison between the CNN-FCM Model with previous models using sensitivity value.

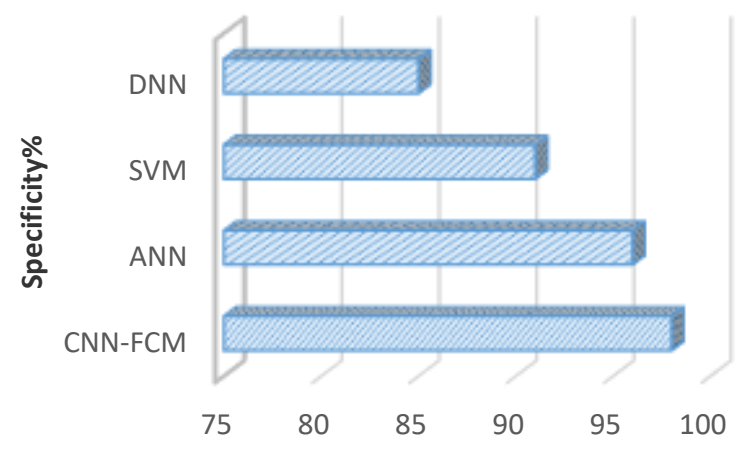

Fig. 8 Comparison between of the CNN-FCM Model with previous models using specificity value.

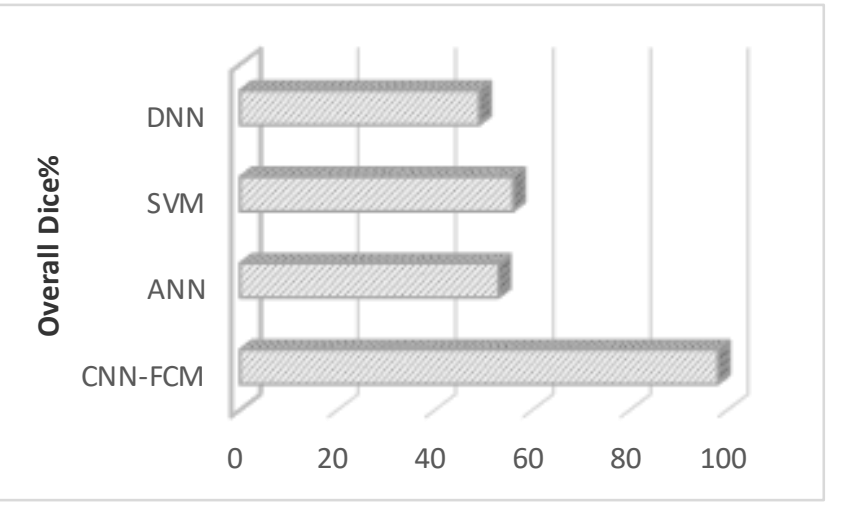

Fig.9 Comparison between of the CNN-FCM Model with previous models using overall dice value.

The figure. 9 represents the improvement of the overall dice value using CNN-FCM model compared with ANN, SVM and DNN models. The proposed model gives the best overall dice value better than all existing models.

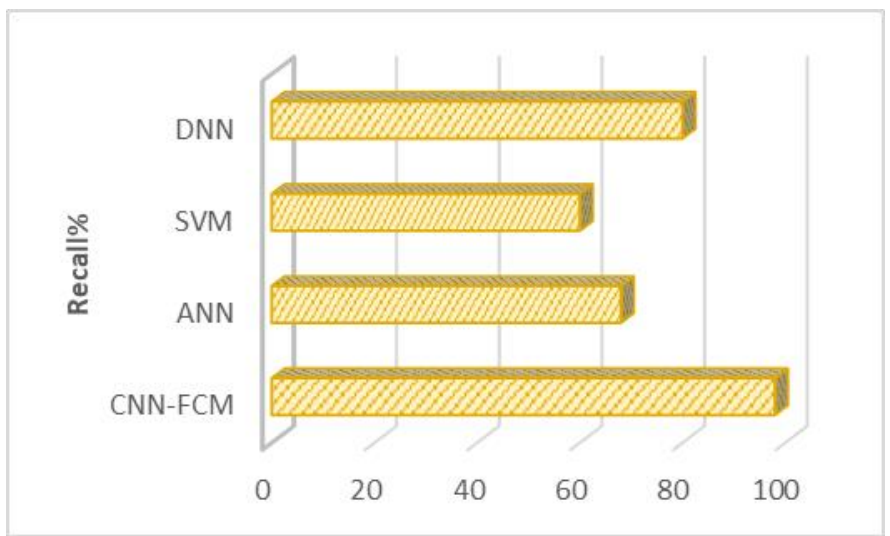

Fig.10 Comparison between of the CNN-FCM Model with previous models using recall value.

Figure.10 described the improvement of the recall value using CNN-FCM model compared with ANN, SVM and DNN models. The proposed model produced a higher recall value better than all existing models.

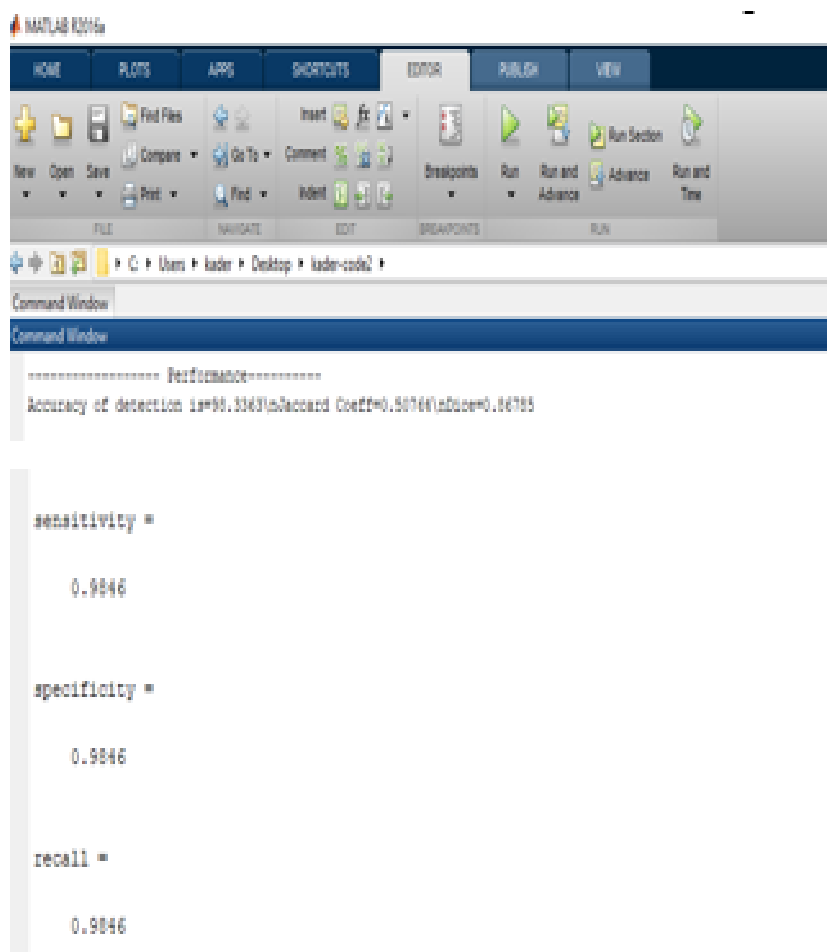

Fig.11. the Overall Performance of the CNN-FCM Model Using Matlab Environment.

Figure.11 shows the improvement of the overall performance of the proposed CNN-FCM model using accuracy, sensitivity, specificity, general dice and recall values. The proposed models achieved the best results for all the values compared with existing models. This proved that the proposed model is very useful and be able to apply in computer aide techniques. 


\section{CONCLUSION}

In this work, we proposed a new model based on a convolution neural network with fuzzy c-mean named as CNN-FCM model to identify brain tumor and analysis using MR BRATS2013 database. The proposed model gives the best results about tumor as identify an area in fig.4. It also achieved the best accuracy, sensitivity, specificity, overall dice and recall values as shown in the fig.5, 6, 7, 8 and 9. The general performance of the model proposed is depicted in fig.10; it shows that the model produced the best performance according to the values. Compared with ANN, SVM and DNN models that were published in top journals, our proposed model gives a better overall performance in all values. According to the high performance of the CNN-FCM model, we strongly recommended it to use in computer aided in medical diagnosis techniques.

\section{ACKNOWLEDGMENT}

I would like to thank "the Natural Science Foundation of China under Grant (51377045 and Grant 31400844), This work was also supported by the Specialized Research Fund for the Doctoral Program of Higher Education under Grant (20121317110002) and Grant (20131317120007).

\section{References}

[1] Moise D, Madhusoodanan S. "Psychiatric symptoms associated with brain tumors: a clinical enigma. CNS Spectr" 11:28-31.2006.

[2] Amin J et al. "Detection of brain tumor based on features fusion and machine learning". J Ambient Intell Humaniz Comput 1-17.2018.

[3] Gooya A et al. "GLISTR: glioma image segmentation and registration”. IEEE Trans Med Imaging 31:19411954.2012

[4] Singhal AB et al. "Ischemic stroke: basic pathophysiology and neuroprotective strategies". In: Acute ischemic stroke. Springer, pp 1-24.2011.

[5] Maier O et al. "Extra tree forests for sub-acute ischemic stroke lesion segmentation in MR sequences". J Neurosci Methods 240:89-100.2015.

[6] Menze $\mathrm{BH}$ et al. "The multimodal brain tumor image segmentation benchmark (BRATS)". IEEE Trans Med Imaging 34:1993.2015.

[7] Prastawa $M$ et al. "A brain tumor segmentation framework based on outlier detection". Med Image Anal 8:275-283.2004.

[8] Reza S, Iftekharuddin K "Improved brain tumor tissue segmentation using texture features. In: Proceedings MICCAI BraTS (brain tumor segmentation challenge), pp 27-30.2014.
[9] Kleesiek J et al." Ilastik for multi-modal brain tumor segmentation." In: Proceedings MICCAI BraTS (brain tumor segmentation challenge), pp 12-17.2014.

[10] Havaei $\mathrm{M}$ et al. "Brain tumor segmentation with deep neural networks". Med Image Anal 35:18-31.2017.

[11] Pereira S et al. "Brain tumor segmentation using convolutional neural networks in MRI images". IEEE Trans Med Imaging 35:1240-1251.2016.

[12]Havaei $M$ et al. "A convolutional neural network approach to brain tumor segmentation". In: International workshop on brain lesion: glioma, multiple sclerosis, stroke and traumatic brain injuries, pp 195-208.2015.

[13] Dvor “a 'k P, Menze B" Local structure prediction with convolutional neural networks for multimodal brain tumor segmentation". In: International MICCAI workshop on medical computer vision, pp 59-71.2015

[14]Fan J et al. "BIRNet: brain image registration using dual supervised fully convolutional networks". Med Image Anal 54:193-206.2019.

[15] Farahani K et al." Brats 2014 challenge manuscripts". http:// www.braintumorsegmentation.org . Accessed 23 July 2019.

[16] Mahapatra D, Bozorgtabar B, Garnavi R. "Image superresolution using progressive generative adversarial networks for medical image analysis". Comput Med Imaging Graph; 71:30-9.2019.

[17]Ren S, Jain DK, Guo K, Xu T, Chi T. "Towards efficient medical lesion image super-resolution based on deep residual networks". Signal Process Image Commun; 75:110.2019 .

[18] Lu X, Huang Z, Yuan Y. "MR Image super-resolution via manifold regularised sparse learning". Neurocomputing; 162:96-104.2015

[19]Zeng K, Zheng H, Cai C, Yang Y, Zhang K, Chen Z. "Simultaneous single-and multi-contrast super-resolution for brain MRI images based on a convolutional neural network". Comput Biol Med; 99:133-41.2018.

[20] Velasco NF, Rueda A, Santa Marta C, Romero E. "A sparse Bayesian representation for super-resolution of cardiac MR images". Magn Reson Imaging; 36:7785.2017.

[21]Das V, Dandapat S, Bora PK. "A novel diagnostic information based framework for super resolution of retinal fundus images". Comput Med Imaging Graph; 72:22-33.2019.

[22] Saad NM, Bakar SARSA, Muda AS, Mokji MM. Review of brain lesion detection and classification using neuroimaging analysis techniques. Journal Technology; 74(6). 2015.

[23] Sachdeva J, Kumar V, Gupta I, Khandelwal N, Ahuja CK. A package-SFERCB Segmentation, feature extraction, reduction and classification analysis by both SVM and ANN for brain tumors. Appl Soft Comput 2016; 47:15167. 
[24] Suresh S, Saraswathi S, Sundararajan N. "Performance enhancement of extreme learning machine for multicategory sparse data classification problems". Eng Appl Artif Intell; 23(7):1149-57.2010.

[25] Mallick PK, Ryu SH, Satapathy SK, Mishra S, Nguyen GN, Tiwari P. "Brain MRI image classification for cancer detection using deep wavelet autoencoder-based deep neural network". IEEE Access; 7:46278-87.2019.

[26] AhmedS, ET al. "Brain tumor classification for MR images using transfer learning and fine-tuning". Comput Med Imaging Graph; 75:34-46.2019.

[27] Deepak S, Ameer PM. "Brain tumor classification using deep CNN features via transfer learning". Comput Biol Med; 111:103345.2019.

[28] Selvapandian A, Manivannan K. "Fusion based glioma brain tumor detection and segmentation using ANFIS classification". Comput Meth Programs Biomed; 166:338.2018 .

[29] Pan Y, Huang W, Lin Z, Zhu W, Zhou J, Wong J, Ding Z. "Brain tumor grading based on neural networks and convolutional neural networks". 37th Annual International Conference of the IEEE Engineering in Medicine and Biology Society (EMBC). IEEE; 2015. p. 699-702.2015.

[30]M. Prastawa et al., "A brain tumor segmentation framework based on outlier detection," Med. Image Anal., vol. 8, no. 3, pp. 275-283, 2004.

[31]B.H.Menzeetal "A generative model for brain tumor segmentation in multi-modal images," in Medical Image Computing and Comput.AssistedInterventionMICCAI2010. New York: Springer, pp. 151-159.2010.

[32]D. Kwon et al., "Combining generative models for multifocal glioma segmentation and registration," in Medical Image Computing and Comput.-Assisted Intervention-MICCAI. New York: Springer, pp. 763770.2014.

[33]A. Gooya et al., "GLISTR: Glioma image segmentation and registration," IEEE Trans. Med. Imag., vol. 31, no. 10, pp. 1941-1954, Oct. 2012.

[34]B. Menze et al., "The multimodal brain tumor image segmentation benchmark (BRATS)," IEEE Trans. Med. Imag., vol. 34, no. 10, pp. 1993-2024, Oct. 2015.

[35] S. Bauer, L.-P. Nolte, and M. Reyes, "Fully automatic segmentation of brain tumor images using support vector machine classification in combination with hierarchical conditional random field regularization," in Medical Image Computing and Comput.-Assisted InterventionMICCAI2011.newYork: Springer, pp. 354-361.2011

[36]R. Meier et al., "Appearance-and context-sensitive features for brain tumor segmentation," in MICCAI Brain Tumor Segmentation Challenge (BraTS), pp. 20-26.2014.

[37] Mohan, J., V. Krishnaveni, and Yanhui Guo. "A survey on the magnetic resonance image denoising methods." Biomedical Signal Processing and Control 9: 56-69.2014.
[38] Manjn, Jos V."MRI preprocessing." Imaging Biomarkers. Springer, Cham. 53-63.2017.

[39] Krizhevsky, A.; Sutskever,I.; Hinton, G.E.: "Image net classification with deep convolutional neural networks". In: Advances in Neural Information Processing Systems, pp. 1097-1105.2012.

[40] Farzam Kharajinezhadian and Saeid Rashidi "A Multimodal Authentication for Biometric Verification System using Palm prints and Fingers" WSEAS Transactions on Signal Processing, pp. 129-141, Volume 15, 2019.

[41]A. Mehidi, M. Mimi, M. Bentoumi, A. Taleb Ahmed, "Contrast Enhancement and Detection of Micro calcifications Mammary Using Hidden Markov Chains "International Journal of Biology and Biomedical Engineering, pp 84-93, Volume 12, 2018.

\section{Authors Contributions:}

Isselmou Abd El Kader: Writing, simulation and analysis.

Guizhi Xu: Review\& editing.

Zhang Shuai: Technical Review.

Sani Saminu: Language Review.

Imran Javaid: writing methodology.

Isah Salim Ahmad: Formatting.

\section{Sources of funding for research presented in a scientific article or scientific article itself:}

This work was supported by "the Natural Science Foundation of China under Grant (51377045 and Grant 31400844), This work was also supported by the Specialized Research Fund for the Doctoral Program of Higher Education under Grant (20121317110002) and Grant (20131317120007)."

\section{Creative Commons Attribution License 4.0 (Attribution 4.0 International, CC BY 4.0)}

This article is published under the terms of the Creative Commons Attribution License 4.0 https://creativecommons.org/licenses/by/4.0/deed.en US 